\title{
Équations de l'écoulement non lent en milieu perméable
}

\author{
Application au calcul des filtres à houle*
}

\author{
Equations of relatively rapid flow through permeable matter \\ Application to the calculation of "wave filters" * \\ English synopsis p. 96
}

La mise en œuvre des filtres à houle a posé une série de problèmes nouveaux. Le principal d'entre eux est celui de la détermination des dimensions à donner aux filtres pour obtenir le taux d'amortissement désiré.

Une première méthode consiste à faire le bilan énergétique du phénomène, c'est-à-dire à évaluer la perte d'énergie due au frottement de l'eau contre les parois ou les obstacles constituant le filtre et à déduire du taux de déperdition d'énergie le taux d'amortissement des houles.

Cette méthode, dont on concoil aisément le principe, présente diverses modalités d'application suivant la manière dont on évalue la force de frottement, donc la déperdition d'énergie. Le mode d'évaluation le plus simple consiste à admettre l'existence d'un frottement visqueux constamment proportionnel à la vitesse. La détermination du coefficient de proportionnalité (c'està-dire de la viscosité apparente) est assez délicate, mais, une fois cette difficulté surmontée, les intégrations se font aisément et les résultats se laissent exprimer par des formules simples et pratiques que nous retrouverons d'ailleurs à la fin de cette note. Natons simplement que ces formules expriment que le taux d'amortissement est proportionnel à la fréquence des houles traversant le filtre.

Cette méthode a été perfectionnée par

* (Voir l'article sur le filtre à houle, dans le $n^{\circ} 3$ de In Houlle Blanche, 1948.)
O'Bracy (1) par la prise en compte du fait que le mouvement de la houle n'est pas continu mais pulsatoire. Ainsi les formules donnant la résistance visqueuse ne sont pas les mêmes que pour un mouvement permanent. La modification apportée par ces considérations se traduit en particulier par le fait que Ie taux d'amortissement devient proportionnel à la puissance $3 / 2$ de la fréquence.

La contradiction à laquelle on aboutit ainsi n'est d'ailleurs qu'apparente. La validité de l'une ou l'autre théorie dépend de la valeur du paramètre $r$ :

$$
r=\frac{k e^{2}}{\nu}
$$

où $k$ est la fréquence angulaire de la houle,

e une longueur caractérisant l'écartement des parois ou des obstacles constituant le filtre,

v la viscosité (réelle ou apparente) suivant les cas).

Pour de petites valeurs de $r$ la première méthode est valable.

Pour de grandes valeurs de $r$, c'est la méthode de O'Brren qui doit être appliquée.

Nous n'insistons pas plus sur cette discussion

(1) O. Brien. - On the effect of wall friction on gravily waves. Transactions of the American geophysical Union 1942 , p. 84. Chronologiquement c'est cet article qui a servi de base a nos premières recherches théoriques sur le comportement des filtres à plaques parallèles. 
qui sort du cadre de cet article et que nous n'avons évoquée qu'à titre indicatif.

Enfin un troisieme mode d'application de la mème méthode qui s'applique essentiellement aux filtres à grande échelle où le frottement n'est plus visqueux mais turbulent, consiste à tenir compte de la loi exacte liant le frottement a la vitesse. On sait que cette loi, rarement comnue avec précision, peut varier de la simple proporlionnalité à la fonction quadratique.

Cette façon de procéder est, scmble-t-il, la plus «consciencieuse » des trois. Mais, étant donné la complexité des lois du frottement turbulent, elle nécessite des intégrations numériques et on ne peut exprimer les résultats obtenus par des formules pratiques et simples comme dans les cas précédents.

On voit que la méthode du « bilan énergétique $\gg$ est extrêmement souple et peut s'adapter à une très large gamme de filtres. Elle a rendu de très grands services et nous a permis de dimensionner convenablement les premiers filtres que nous avons construits. Cependant elle présente quelques défauts. 'Tout d'abord, c'est une méthode empirique dont la validité n'a jamais été solidement établie. Rien ne prouve que l'action des forces de frottement se traduise uniquement par un amortissement. Ny a-t-il pas également une distorsion des houles, la création de parasites nuisibles?

Enfin, si cette méthode, pour imprécise qu'elle soit, donne des indications convenables sur lo taux d'amortissement, elle laisse tout ignorer sur le mécanisme interne de l'amortissement, on ne sait ni en quoi le mouvement des houles dans le filtre diffère du mouvement des houles libres ni l'importance eventuelle que peut avoir cette différence.

C'est en cherchant à combler ces lacunes et à avoir des vues plus précises sur les phénomènes hydrodynamiques accompagnant l'amortissement des houles que nous avons été conduits à établir la théorie qui suit.

Signalons en terminant qu'on ne tient pas compte de l'amortissement propre (de distorsion interne). En effet, dans la quasi-totalité des cas, cette cause d'amortissement est négligeable à côté de celle due au filtre.

Le milieu perméable classique est défini, au point de vue de l'hydrodynamique théorique, par la propriété suivante :

Chaque particule d'eau traversant le milieu (que nous supposons immobile) est soumise de la part de celui-ci à une force (de frottement vis- queux) proportionnelle à sa vitesse et de direction opposée.

Nous ne précisons pas plus cette définition, qui est bien connue.

Les ćcoulements en milieu perméable (terres, sables, graviers, etc.) sont en général très lents. C'est pourquoi la thérie classique néglige leffet des forces d'inertie, La force de frottement cquilibre à elle seule l'eflet des forces extérieures et du gradient de pression, ce qui fait que, dans le cas des écoulements gravitaires, par exemple, la vitesse est tout simplement dirigée suivant l'opposé du gradient de hauteur piézométrique.

L'hypothèse de la lenteur de l'écoulement est presque toujours justifiée ne serait-ce que parce que des écoulements un peu rapides ne peuvent se concevoir que dans des milieux poreux à larges circulations (c'est-à-dire à grande perméabilité) et qu'ainsi, le plus souvent, ils sortent du domaine des écoulements viscueux.

Cependant l'étude d'équations plus complètes fenant compte des forces d'inertie n'est pas sans intérêt.

En effet, l'action du froltement turbulent est identique à celle du frottement visqueux, et pour beaucoup de problemes il est - heureusement - admissible de remplacer la loi quadratique ou autre du frottement turbulent par une loi linéaice en introduisant une viscosité fictive de valeur appropriée. Les résultats ainsi obtenus sont certes moins précis et leur interprétation risque d'être plus délicate, mais ils ont l'avantage essentiel d'exister et d'être obtenus grâce à une théorie relativement simple.

Rappelons que, dans ce qui suit, si nous considérons le fluide comme visqueux en ce qui concerne son «frottement» avec le massif, par contre nous le considérons comme parfait en ce qui concerne les frottements internes.

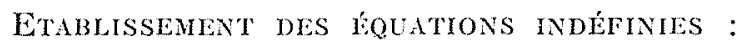

Les équations générales du mouvement irrotationnel d'un fluide parfait incompressible peuvent s'écrire en coordonnées d'Euler :

$$
\operatorname{grad} \frac{p}{p}+\operatorname{grad} \frac{\partial \varphi}{\partial t}+\operatorname{grad} \frac{p^{2}}{2}-\overline{\mathrm{F}}=0,
$$

$p$ étant la pression, p la densité, o le potentiel harmonique des vitesses, $v$ la vitesse et $\bar{F}$ la force appliquée à l'unité de masse du liquide.

Dans le cas qui nous occupe, $\bar{F}$ est divisé en deux composantes :

$1^{\circ}$ Les forces extérieures $F_{e}$ (gravité, force cen- 
trifuge, etc.), dont nous supposerons qu'elles admettent un potentiel :

$$
\mathrm{U}\left(\mathrm{F}_{e}=\operatorname{grad} \mathrm{U}\right) \text {. }
$$

$2^{\circ}$ Les forces de frottement visqueux $\mathrm{F}_{v}$ qui, par hypothèse, sont de la forme :

$$
\mathrm{F}_{v}=-\lambda_{v}=-\lambda \operatorname{grad} \varphi,
$$

$\lambda$ étant une constante positive.

Si nous portons ces valeurs dans (1), nous obtenons l'écuation :

$$
\begin{aligned}
\operatorname{grad} \frac{p}{\rho}+\operatorname{grad} \frac{\partial \varphi}{\partial t}+\operatorname{grad} \frac{v^{2}}{2} & -\operatorname{grad} \mathrm{U} \\
& +\lambda \operatorname{grad} \rho=0,
\end{aligned}
$$

soil :

$$
\operatorname{grad}\left[\frac{p}{\rho}+\frac{\partial \varphi}{\partial t}+\frac{v^{2}}{2}-\mathrm{U}+\lambda \varphi\right]=0 .
$$

Soit encore l'équation équivalente :

$$
\frac{p}{i}+\frac{\partial \varphi}{\partial t}+\frac{v^{2}}{2}-\mathrm{U}+\lambda \varphi=\text { cte. }
$$

(La constante pouvant aisément être prise égale à zéro en donnant à $U$ une détermination convenable.)

Dans le cas particulier ou les forces exterieures se réduisent à la pesanteur, nous avons :

$$
\mathrm{U}=-g y
$$

(Oy était un axe vertical ascendant), doù l'équation :

$$
\frac{p}{p}+\frac{\partial \varphi}{\partial t}+\frac{v^{2}}{2}+g y+\lambda \varphi=\text { cte. }
$$

L'équation (3) est une généralisation de l'équation de Bernouilli pour les mouvements non lents et non permanents en milieu perméable. Souvent les termes de l'ordre du carré des vitesses seront négligables. On pourra alors utiliser l'équation simplifiée ci-dessous qui a le gros avantage d'être linéaire :

$$
\frac{p}{p}+\frac{\partial \varphi}{\partial t}+g y+\lambda \varphi=c t e .
$$

C'est cette dernière équation que nous utiliserons pour l'étude des houles, étant donné que nous nous limiterons aux théories du premier ordre.

\section{Application a L'ÉtUde DE L'AMORTISSEMLYT DES HOULES DANS LES FILTRES NEYRPIC}

Les filtres à houle constituent précisément des massifs perméables dans lesquels l'eau conserve des vitesses et des accélérations non né- gligeables tout en subissant un freinage appréciable.

Dans ce qui suit, nous nous limiterons aux problemes plans à deux dimensions et aux houles en profondeur constante. Nous nous reporterons à un système d'axes rectangulaires $O x$ horizontal et $O y$ vertical ascendant placés dans le plan du mouvement. Ox sera de plus supposé confondu avec la surface libre au repos.

De plus, on désignera par :

$h$ la profondeur (constante) de l'eau,

$\mathrm{L}$ la «longueur d'onde » avec:

$$
\begin{aligned}
& m=\frac{2 \pi}{\mathrm{L}} \\
& \mathrm{T}=\text { la période avec : } \\
& k=\frac{2 \pi}{\mathrm{T}}
\end{aligned}
$$

$\eta(x)=$ l'équation de la surface libre.

Nous sommes ainsi amenés au problème suivant :

Trouver une fonetion $\varphi$, représentant un mouvement ayant l'allure de celui d'une houle amortie et salisfaisant aux conditions suivantes :

$$
\begin{array}{ll}
1^{\prime \prime} \Delta_{x, y} \varphi=0 & \\
2^{\circ} \frac{\partial \varphi}{\partial y}=0 & \text { pour } y=-\ldots h \\
3^{\circ} p=\text { cte } & \text { pour } y=\eta(x)
\end{array}
$$

En transformant la dernière condition par des substitutions bien connues et en tenant compte de l'équation (4), nous l'obtenons sous la forme:

$$
\frac{\partial \hat{\varphi}}{\partial t}+\lambda \varphi+g \int^{\prime} \frac{\partial \varphi}{\partial y} d t=0
$$

Nous allons montrer que notre problème est rigoureusement résolu par la fonction :

$$
\begin{array}{r}
\varphi=\frac{a_{0} k e^{-\beta m x}}{m \operatorname{sh} m h} \cos \beta m(y+h) \operatorname{ch} m(y+h) \\
\sin (m x-k t) \\
+\frac{a_{0} k e^{-\beta m x}}{m \operatorname{sh} m h} \sin \beta m(y+h) \operatorname{sh} m(y+h) \\
\cos (m x-k t)
\end{array}
$$

On s'assure aisément que la première condition est satisfaite en remarquant que ost la partie réelle de la fonction :

$$
Z=\frac{a_{0} k}{m \operatorname{sh} m h} e^{-\beta m\left(z^{i} i h\right)} \sin (m z-k t+i m h)
$$

II est également facile de voir que la deuxième relation est satisfaite. 
Nous allons maintenant chercher si la troisième relation peut être satisfaite, pour cela nous l'écrivons en y faisant les substitutions et les transformations convenables, elle se met sous la forme :

$\lceil\lambda \sin \beta m h \operatorname{sh} m h-k \cos \beta m h \operatorname{ch} m h$

$$
\begin{gathered}
-\beta g \frac{m}{l} \sin \beta m h \operatorname{ch} m h \\
\left.+\frac{g m}{k} \cos \beta m h \operatorname{sh} m h\right] \cos (m x-k t)
\end{gathered}
$$

$+[\lambda \cos \beta m h \operatorname{ch} m h+k \sin \beta m h \operatorname{sh} m h$

$$
\begin{aligned}
& -\beta g \frac{m}{k} \cos \beta m h \operatorname{sh} m h \\
& \left.-\frac{g m}{k} \sin \beta m h \operatorname{ch} m h\right]=0 .
\end{aligned}
$$

On en déduit les deux conditions simultances :

$\lambda k \sin \beta m h \operatorname{sh} m h-k^{2} \cos \beta m h \operatorname{ch} m h$

$=g m \beta \sin \beta m h \mathrm{ch} m h-g m \cos \beta m h \operatorname{sh} m h$

$\lambda k \cos \beta m h \operatorname{ch} m h+k^{2} \sin \beta m h$ sh $m h$

$=g m \beta \cos \beta m h$ sh $m h+g m \sin \beta m h$ ch $m h$

On en déduit aisément que la troisième relation est également satisfaite si l'on a :

$$
k^{2}=m g \text { th } m h \times \frac{1-\beta \frac{\sin 2 \beta m h}{\operatorname{sh} 2 m h}}{1-\frac{\sin ^{2} \beta m h}{\operatorname{ch}^{2} m h}}
$$

$$
\lambda=\beta k \frac{1+\frac{\sin 2 \beta m h}{\beta \operatorname{sh} 2 m h}}{1-\frac{\operatorname{sh} 2 m h}{k \sin 2 \beta m h}}
$$

Ces expressions se simplifient notablement si l: est tres petit, elles deviennent alors (1):

$$
\begin{gathered}
k^{2}=m g \text { th } m h \\
\left(k^{2}=m g \text { pour } h \text { très grand }\right) \\
\lambda=\beta h\left[1+\frac{2 m h}{\operatorname{sh} 2 m h}\right]
\end{gathered}
$$

Les erreurs relatives iutroduites au cours de cette simplification sont inférieures à $\beta^{2}$ (autrement dit à $100 \beta^{2} \%$ ) même pour de très grandes valeurs de $h$.

Afin de permeltre au lecteur de juger de l'importance de cette erreur, nous précisons qu'à la valeur $\beta: 1 / 10$ correspond une diminution de l'amplitude de $50 \%$ pour un parcours d'une longueur d'onde.

Ce système d'équation permet de déterminer $m$ et $\lambda$ quand on connaît $k$ et $\beta$.

Ainsi, connaissant la fréquence et le module d'amortissement $\beta$, on peut déterminer la longueur d'onde et le coefficient de perméabilité. Cette proposition admet un certain nombre de réciproques évidentes.

En portant les valeurs ainsi calculées dans (6), on obtient donc bien une solution qui satisfait à toutes les conditions énoncées.

L'amortissement de $50 \%$ de l'amplitude en une longueur d'onde est déjà très rapide. Pourtant, dans les cas exceptionnels où l'amortissement est encore plus brutal, il peut être nécessaire d'utiliser les formules (9) et (10).

\section{F. Brésel.}

(1) Ce sont les formules obtenues par la toute première des méthodes que nous avons rappelées au début de cette note. 